\title{
Lyme Disease: Giving Voice to the Illness Experience-An Exploratory Descriptive Study
}

\author{
Heidi S. Kulkin, PhD, LCSW-BACS \\ Southeastern Louisiana University \\ 310 West Dakota Avenue \\ SLU 10863 \\ Hammond, Louisiana 70403 \\ USA
}

\begin{abstract}
The purpose of this study was to explore and describe the illness experience of patients suffering with Lyme disease. A survey containing demographic and Likert Scale items (The Illness Experience Survey) was constructed and administered to patients suffering with Lyme disease. Descriptive statistics were analyzed to produce findings that: 1) described the participants in the study, and2) described the participants' illness experience. The study concluded that the pain and suffering that Lyme patients experience is undeniable and that people afflicted with Lyme disease appear to be at-risk of disenfranchisement by society at-large and the mainstream medical community; thus, adversely affecting their illness experience.
\end{abstract}

Keywords: Lyme disease, illness experience, contested illness, disenfranchised grief

\section{Introduction}

Over the last several years, there has been an increase in media attention focusing on Lyme disease and the various disabling effects of the illness. Currently, the Center for Disease Control (CDC, 2019) estimates 30,000 new reported cases of Lyme disease each year based on the standard national surveillance methods. However, the CDC (2019) acknowledges that the actual number of Lyme disease cases may be 10 times higher than the actual number of reported cases. Thus, the actual number of new cases on a yearly basis appears to be closer to 300,000 (CDC, 2019). Based on information reported by the CDC (2019), Lyme disease appears to be spreading in epidemic proportions.

While there has been more media attention focusing on the disease, research on the physical and mental effects of this debilitating disease lags behind. The apparent reason for the lag in research seemingly stems from the controversy surrounding the illness. That is, issues surrounding diagnostic testing for Lyme disease, recognition by the medical community that Lyme disease and chronic Lyme disease exist, and set protocols for treatment of the illness (Ali, Vitulano, Lee, Weiss, \& Colson, 2014; Ferguson, 2012; Hill \& Holmes, 2015; Horowitz, 2017; Johnson, Aylward, \& Stricker, 2011; Rebman, Aucott, Weinstein, Bechtold, Smith, \& Leonard, 2017).

Current literature focusing on Lyme disease describes the disease as a controversial and contested illness (Ali et al., 2014; Ferguson, 2012; Johnson et al., 2011; Rebman et al., 2017). At the present time, there are two opposing viewpoints related to the disease. There are the guidelines set forth by the Infectious Diseases Society of America (IDSA, 2019) and the guidelines set forth by the International Lyme and Associated Diseases Society (ILADS, 2019)(Cameron, Johnson, \& Maloney, 2014; Johnson et al., 2011, Ferguson, 2012; IDSA, 2019; ILADS; 2019; Wormser et al., 2006). The view engendered by IDSA is that Lyme disease is rare, localized only to certain regions, and that chronic infection of the illness is non-existent. On the contrary, ILADS argues that the disease is not rare and, because the disease can be transmitted by rodents, deer, and birds, it can be found world-wide. In addition, ILADS points out that tick bites often go unnoticed and that laboratory testing for the disease often produces inaccurate results. Therefore, the disease may not be diagnosed leading to a persistence of chronic Lyme disease in undiagnosed individuals. Undiagnosed individuals are many times plagued by unexplained or contested disorders/syndromes (e.g., fibromyalgia and chronic fatigue syndrome) with no apparent etiological origin. The controversy and complexities surrounding the illness has placed Lyme disease in the same category as other contested illnesses (e.g., multiple chemical sensitivity and myalgic encephalomyelitis). Contested illnesses are seen as illnesses with no known etiology, no known biological cause, and; thus, no set protocols undergirding treatments for the illnesses (Rebman et al., 2017). As noted by Conrad and Barker (2010), contested illnesses typify a unique type of chronic illness. Main themes in the literature related to contested illnesses are: 1) The struggle for illness legitimacy in medical and social settings, 2) Psychological explanations of the symptoms, which are viewed as dismissive and delegitimizing, and 3) The large degree of doubt and uncertainty experienced by patients living with contested illnesses (Barker, 2002; Hyden \& Sachs, 1998; Nettleton, 2006). 
Due to the nature of contested illnesses and the adversities faced by those afflicted, it is important that clinicians, practitioners, and providers working with patients struggling with Lyme disease, a contested illness, be consciously aware of patients' lived experiences (Rebman et al., 2017). In essence, placing themselves in the shoes of their Lyme patients in order to experience what the patient is experiencing. To this end, clinicians, practitioners, and providers may understand and be more empathic to the plight and needs of their Lyme patients.

The purpose of the current study was to explore and describe the illness experience of patients suffering with Lyme disease. That is, not only the overall/general experiences of patients suffering with Lyme disease, but also the exploration of grief experienced by Lyme patients. This author presupposed that by virtue of Lyme being a contested illness, patients suffering with the disease would not only experience grief as it relates to chronic illness (e.g., loss of independence, loss of old self, loss of physical health, etc.), but also experience disenfranchised grief surrounding their illness. As Doka (2002) describes, disenfranchised grief is characterized when a person has experienced a loss, but the person does not have a "right" to grieve that loss since the loss is not recognized as a legitimate cause of grief. Since there is such conflict in terms of legitimizing contested illnesses, as in the case of Lyme disease, it would follow suit that those experiencing a contested illness would experience disenfranchised grief.

At present, there is a dearth of literature focusing on the experiences faced by Lyme patients and there are no studies exploring or describing grief and/or disenfranchised grief and Lyme patients. Thus, the aim of this study was to fill the gaps in the literature concerning: 1) the overall illness experience of Lyme patients, and 2)the grief and disenfranchised grief of Lyme patients. Moreover, it is imperative that clinicians, practitioners, and providers familiarize themselves with the plight of those who are suffering with Lyme to best help this population of people. This exploratory descriptive study sought to shed light on the experiences of those suffering with Lyme disease. By illuminating the experiences of those suffering with Lyme disease, clinicians, practitioners, and providers may become consciously aware of the perplexities of the situation faced by Lyme patients and may be better equipped to work with these individuals.

\section{Methods}

For this exploratory descriptive study, the author used a convenience sample of participants suffering with Lyme disease. An electronic survey containing a cover letter, demographic items, and Likert Scale statement items (The Illness Experience Survey) was posted in an online Lyme disease support group. The cover letter outlined that ethical approval to carry out the study was obtained through Southeastern Louisiana University's Institutional Review Board Committee (ethical board). Additionally, the cover letter discussed the nature of the study and that if a participant consented to take part in the study, they did so by completing and submitting the survey; thus, giving the author consent to include the participant's survey data in the study.

\subsection{The Survey}

The author constructed a survey which included two sections. One section focused on demographic items, and another section contained Likert Scale statement items.

2.1.1 Demographics. The demographic section of the survey included information such as: age, gender, level of education, whether the participant had been diagnosed by a medical professional with Lyme disease, length of illness, etc. (Table 1), to describe the characteristics of participants in the study.

2.1.2 Likert Scale statements. The Likert Scale statement item section, called The Illness Experience Survey, was based on: 1) the aim of this study,2) current literature available on individuals suffering with Lyme disease and/or chronic illness (Ali et al., 2014; Ferguson, 2012; Horowitz, 2017; Jason \& Reed, 2015; Johnson et al., 2011; Morris \& Sanders, 2018; Nicolai et al., 2018; Ortenblad, Meillier, \& Jonsson, 2018; Rebmanet al., 2017; Siegal, Brown, Devendoff, Collier, \& Jason, 2018; Slightam, Brandt, Jenchura, Lewis, Asch, \& Zulman, 2018; van den Wijngaard, 2015), 3) current literature on grief in general as related to chronic illness (Boss \& Couden, 2002; Gill \& Lowes, 2014), 4), literature on disenfranchised grief (Attig, 2004; Doka, 2002;Gill \& Lowes, 2014; Sobel \& Cowan, 2003), and 5) information gleaned through this author's experiences in speaking to many people suffering with chronic Lyme disease over the course of several years. The list of Likert Scale statement items listed on The Illness Experience Survey is outlined in Table 2.

\subsection{Participants}

The criteria for inclusion in this study were that a participant had to have been diagnosed by a medical professional with Lyme disease and that a participant was 18 years or older. The demographic questions, "Have you been diagnosed by a medical professional with Lyme disease?," and, "Age," were used as screening mechanisms for inclusion in this study. One hundred participants completed the survey; however, nine did not meet criteria for inclusion in the study (n $=8$ were not diagnosed with Lyme disease by a medical professional and $\mathrm{n}=1$ was not 18 years of age or older). 
Thus, there were a total of 91 participants included in this study. Of the 91 participants, $70.4 \%(n=64)$ of the participants reported their age range between 45-64 and an overwhelming majority was female (85.7\%). Demographic information of participants is listed in Table 1.

\section{Results}

\subsection{Data analysis}

Descriptive statistics such as percentages and actual number of responses for each item were analyzed on the demographic section (Table 1) and The Illness Experience Survey (Table 2). The responses in relation to Likert Scale statement items on The Illness Experience Survey (Strongly agree, agree, neither agree nor disagree, disagree, and strongly disagree) were aggregated into the categories of agree, neither agree nor disagree, and disagree for the purpose of summarizing the data for report. Thus, the response of agree incorporated the choice categories of strongly agree and agree, while the response of disagree incorporated the choice categories of disagree and strongly disagree. The choice category of neither agree nor disagree remained unchanged.

\subsection{Item responses}

Table 2 illustrates the statement and item responses on The Illness Experience Survey. Each statement and corresponding responses are shown.

As depicted in Table 2, a majority (three quarters or more) of participants, agreed with most item statements on The Illness Experience Survey. That is, 25 out of 35 statement items elicited agreed responses ranging from $68.1 \%$ to 96.7\% of participants agreeing with the statement item.

There were ten statement items on The Illness Experience Survey that elicited lower percentages of agree responses. Percentage responses with answers of agree for those ten items ranged from $27.5 \%$ to $56.2 \%$. The ten items dealt with: adjusting to life being chronically ill (50.4\% agreed), believing that they will go into remission (45\% agreed), doctors believing their reports concerning their symptoms (42.2\% agreed), treating self with no medical assistance $(37.8 \%$ agreed), spending thousands of dollars only to become sicker (56.2\% agreed), accepting their limitations (42.9\% agreed), making a new life plan since becoming ill (48.9\% agreed), finding support in being a member of an in-person self-help group (27.5\% agreed), sought counseling (50\% agreed), and believing they don't have the right to grieve over their losses related to their illness (39.6\% agreed).

\section{Discussion}

This study gives voice to a population of people suffering with a contested illness, Lyme disease. The results of this study provide insights into the overall/general illness experience and grief experiences (general and disenfranchised) of patients, 18 years and older, who have been diagnosed by a medical professional with Lyme disease. Findings of the study support current literature as well as personal accounts and experiences as shared by patients suffering with Lyme disease with the author over the course of many years.

In terms of the overall/general experiences of those suffering with Lyme disease, much of the literature that is available on the experiences of Lyme patients highlight how Lyme disease, especially chronic Lyme disease (CLD), is not recognized by many in the medical community. As the results of this study (high percentage of agreed responses to items numbered 1, 2, 3, 5, 6, 7, 8, 10, 11, 12, 13, 14, 15, 16, 17, 28, \& 34 on The Illness Experience Survey) indicate alongside the results of other studies conducted on those suffering with Lyme disease and/or other contested illnesses, the lack of illness legitimacy has seemingly led Lyme patients to perceive a sense of alienation and abandonment from society, a sense and the reality that they've lost friendships as a result of the illness, a sense that others don't believe that they are ill, a sense of frustration when others don't believe they are ill, a sense of frustration by the lack of help from the medical community, a sense that cancer is a much more accepted illness, a sense that doctors don't know how to treat the illness, a sense that they live in fear that they will become sicker, a sense that it just takes too much energy to try and explain the disease to others, a sense of uncertainty about recovery and progression of the illness, a sense that doctors are reluctant to believe patients' report of symptoms, and an overall lack of empathy [by others] concerning their plight (Ali et al., 2014; Drew \& Hewitt, 2006; Mechanic \& Meyer, 2000; Nettleton, Watt, O’Malley, \& Duffy, 2005; Nettleton, 2006; Rebman et al., 2017).

Since Lyme disease and other contested illnesses aren't “medically sanctioned” diseases/illnesses, they aren't typically socially sanctioned diseases and/or illnesses. The lack of legitimacy further complicates the suffering of Lyme patients in that the questionability of the illness precludes Lyme patients the "right" to enter into the socially sanctioned "sick role" (Rebman et al., 2017). The sick role is a role that is assumed by a person who is ill to replace his or her "normal roles." The sick role is characterized as a type of social role where by certain exemptions, rights, and obligations are adopted by the person who is ill. 
Society serves to shape and sanction the exemptions, rights, and obligations afforded to those in the sick role (Fox, 1989). In contrast to patients with Lyme disease, people with socially sanctioned illnesses such as cancer and AIDS are readily accepted by society in adopting the sick role.

Delegitimation of the illness, serves to further complicate the illness experience by affecting the Lyme patient's "right" to grieve over their losses. That is, all of the losses they have experienced as a result of having the illness (e.g., physical health, sense of self, independence, lifestyle change, etc.). If a patient does not have a societally sanctioned illness, the losses they suffer are not seen as legitimate losses. While patients that are societally sanctioned as chronically ill experience much of the same losses (Nicolai et al., 2018) that Lyme patients suffer (e.g., physical health, sense of self, independence, etc.), Lyme patients' losses, by virtue of Lyme being classified as a contested and controversial illness, appear to result in disenfranchised grief on the part of Lyme patients. As Doka (1989) discusses, the feelings usually associated with grief such as anger, guilt, sadness, and depression are intensified when the grief is not validated/socially recognized. When a person's grief is disenfranchised, that person tends to become isolated. As seen in this study, 92.3\% of participants reported feeling very isolated. Additionally, participants reported a sense of feeling that the losses they had suffered as a result of Lyme disease weren't grief-worthy because it wasn't a death. This finding is discussed in the literature (Gill \& Lowes, 2014; Thornton \& Zanich, 2002). That is, non-death related losses are viewed as less intense than death related losses. Thus, those suffering from a disenfranchised loss appear to be at greater risk for failing to seek out support because they believe that no one is available and that they don't necessarily deserve support (Thornton \& Zanich, 2002). In summary, Doka (2002), explicates that disenfranchised grief occurs when a person experiences a loss, but the person feels that they do not have the "right" to grieve the loss since the loss is not considered a legitimate cause of grief. As reported in this study, almost $40 \%$ of participants agreed that they believed that they did not have the right to grieve over the losses that they were suffering as a result of having Lyme disease.

Embedded in The Illness Experience Survey were several statement items dealing with how Lyme disease patients cope with their illness as part of their overall/general experience. As outlined by Rebman et al. (2017), participants in their study reported incorporating coping strategies such as stubbornness, challenging boundaries, accepting limitations, accepting things for the way they are, and making a new life plan since becoming ill (pp. 538-539). In the current study, $68.1 \%$ of participants reported employing stubbornness as a way to combat their disease, $42.9 \%$ reported accepting that things were different, and $48.9 \%$ reported making a new life plan since becoming ill. Additional coping statement items included on The Illness Experience Survey included questions regarding finding support in online groups geared to Lyme disease and chronic illness (85.7\% agreed), finding support in face-to-face self-help groups (27.5\% agreed), spending time on symptom management (78\% agreed), and attending counseling (50\% agreed). Noteworthy is that the additional coping statement items were developed based on this author's numerous discussions, over the course of many years, with patients suffering with Lyme disease.

While the current study certainly adds to the literature regarding the experiences of those suffering with Lyme disease, still much is to be done. Research that is available in relation to the suffering Lyme patients' experience and the controversies surrounding the illness paints a bleak picture for Lyme suffers (Ali et al., 2014; Ferguson, 2012; Hill \& Holmes, 2015; Johnson et al., 2011; Rebman et al., 2017; van den Wijngaard et al., 2015). Future work is warranted on: 1) the examination of protocols and treatments to help alleviate the suffering of Lyme patients, 2) the examination of updated diagnostic measures for Lyme disease that produce accurate results, and 3) the examination of the medical and societal construction of the disease in order to eradicate the stigma, myths, and controversial/contested milieu surrounding the illness. The overall implication is that Lyme disease, as an illness, must be legitimized by the mainstream medical community in order to change the way in which the disease is viewed societally, even globally.

\subsection{Limitations}

Although this study sheds much light on the experiences of patients suffering with Lyme disease, the limitations of the inquiry must be discussed. A convenience sample of non-randomly assigned participants was recruited through an online Lyme support group. Therefore, the sample of participants may not be completely representative of all persons living with Lyme disease. Future studies may wish to incorporate larger randomly selected samples using explanatory design methods with the use of inferential based statistical analyses to test significant mean differences and/or relationships between demographic information and the illness experience on the whole. In this regard, analysis of results would lend itself to generalization to the population at-large.

\section{Conclusions}

Although there has been more media attention given to Lyme disease over the past few years, there is still much to do in the area of research on the physical and mental aspects of the disease. 
Until this illness becomes uncontested and socially/medically sanctioned, Lyme patients will most likely remain in their current plight, suffering without much hope of a cure or at best treatments to control the disease and the symptoms manifested as a result of the illness. It is evident that Lyme patients, clinicians, practitioners, and providers, as well as society at-large would benefit from a better understanding of the disease and the disease process. Specifics concerning changes in policies surrounding the disease and changes to diagnostic testing criteria, as well as changes in the way epidemiologic statistics are gathered are much needed. It is only after the aforementioned items occur that we may find actual treatment methodologies and protocols to combat Lyme disease, and hence, elucidate disease prognosis. Lastly, it is immensely evident that more evidence-based research should be conducted, brought to the forefront, and emphasized in order to facilitate the improvement of care to patients suffering with Lyme disease.

Table 1. Participant demographic information $(N=91)$

\begin{tabular}{|c|c|c|}
\hline Demographic question & $\mathrm{n}$ & $\%$ \\
\hline \multicolumn{3}{|l|}{ Age (by range): } \\
\hline $18-24$ years & 1 & 1.1 \\
\hline $25-34$ & 10 & 10.99 \\
\hline $35-44$ & 14 & 15.38 \\
\hline $45-54$ years & 35 & 38.46 \\
\hline $55-64$ years & 29 & 31.87 \\
\hline $65+$ years & 2 & 2.2 \\
\hline \multicolumn{3}{|l|}{ Gender: } \\
\hline Female & 78 & 85.7 \\
\hline Male & 13 & 14.3 \\
\hline \multicolumn{3}{|l|}{ Level of education: } \\
\hline Less than high school degree & 1 & 1.10 \\
\hline High school degree or equivalent (GED) & 6 & 6.59 \\
\hline Some college, but no degree & 22 & 24.18 \\
\hline Associate degree & 16 & 17.58 \\
\hline Bachelor degree & 27 & 29.67 \\
\hline Graduate degree & 19 & 20.88 \\
\hline \multicolumn{3}{|l|}{ Identify as having chronic Lyme disease (CLD): } \\
\hline Yes & 89 & 99.89 \\
\hline No & 1 & 1.1 \\
\hline \multicolumn{3}{|c|}{ Identify as having Post Treatment Lyme Disease Syndrome (PTLDS): } \\
\hline Yes & 77 & 85.56 \\
\hline No & 13 & 14.44 \\
\hline \multicolumn{3}{|l|}{ Health on a scale from 1 (best) to 10 (worst): } \\
\hline 1 & 1 & 1.11 \\
\hline 2 & 2 & 2.22 \\
\hline 3 & 5 & 5.56 \\
\hline 4 & 7 & 7.78 \\
\hline 5 & 11 & 12.22 \\
\hline 6 & 16 & 17.78 \\
\hline 7 & 22 & 24.44 \\
\hline 8 & 10 & 11.11 \\
\hline 9 & 9 & 10.00 \\
\hline 10 & & \\
\hline \multicolumn{3}{|l|}{ Years been ill (by range): } \\
\hline $1-5$ years & 22 & 24.18 \\
\hline $6-10$ years & 18 & 19,78 \\
\hline $11-15$ years & 12 & 13.19 \\
\hline $16-20$ years & 12 & 13.19 \\
\hline More than 20 years & 27 & 29.67 \\
\hline \multicolumn{3}{|c|}{ Pre-existing conditions that could explain your current symptoms: } \\
\hline Yes & 10 & 10.99 \\
\hline No & 81 & 89.01 \\
\hline
\end{tabular}

Currently working: 
Yes

No

Currently confined to your bed:

Yes

No

Symptoms currently affect your daily life:

Yes

No

Symptoms ever affect your daily life:

Yes

No

In total, out-of-pocket amount (dollars) spent on treatment (by range):

$\$ 0$

Up to $\$ 1,000$

Up to $\$ 2,000$

Up to $\$ 3,000$

$\$ 4,000-10,000$

$\$ 11,000-15,000$

$\$ 16,000-20,000$

$\$ 21,000-50,000$

$\$ 51,000-75,000$

$\$ 76,000-100,000$

Over $\$ 100,000$

Do you have medical insurance:

Yes

No

If you have medical insurance - Lyme treatments covered:

Yes

No

N/A

If you have medical insurance - lab tests related to Lyme covered:

Yes

No

N/A
34

57

17

74

88

3

90

0

2

2

6

3

15

8

10

20

9

3

12

85

6

44

30

8

60

21

8
37.36

62.64

18.68

81.32

96.70

3.30

100.00

0.00

2.22

2.22

6.67

3.33

16.67

8.89

11.11

22.22

10.00

3.33

13.33

93.41

6.56

48.35

42.86

8.79

67.42

23.60

8.99

( $n=$ the participants who answered the question)

Table 2. Survey questions and response results - The Illness Experience Survey

Questions

Agree (n)

Neither agree nor disagree (n)

Disagree (n)

1. It gets to where I feel no one will listen to me.

2. I feel very isolated.

3. I feel that people don't believe I'm sick.

4. I have adjusted to life being chronically ill.

5. I become frustrated when people don't believe that I'm ill.

6. I believe having cancer is much more accepted than having Lyme disease.

7. This disease takes too much energy to explain to people.

8. I've lost friendships during my illness.

9. I grieve over the loss of my old self.

10. I become frustrated because there is an absence of available, established treatment, and disease prognosis from
(84) $92.3 \%$

(84) $92.3 \%$

(75) $84.3 \%$

(55) $50.4 \%$

(72) $79.1 \%$

(88) $96.7 \%$

(86) $94.5 \%$

(78) $85.7 \%$

(86) $94.5 \%$

(87) $95.6 \%$
(4) $4.4 \%$

(1) $1.1 \%$

(9) $10.1 \%$

(13) $14.3 \%$

(13) $14.3 \%$

(2) $2.2 \%$

(2) $2.2 \%$

(5) $5.5 \%$

(3) $3.3 \%$

(3) $3.3 \%$
(3) $3.3 \%$

(6) $6.6 \%$

(5) $5.6 \%$

(23) $25.3 \%$

(6) $6.6 \%$

(1) $1.1 \%$

(3) $3.3 \%$

(8) $8.8 \%$

(2) $2.2 \%$

(1) $1.1 \%$ 
the health care system in terms of my illness.

11. I don't know what the future holds in terms of my being able to recover from this illness.

12. I believe I will go into remission one day.

13. I don't know what to expect in terms of how my illness will progress (what it will look like).

14. I become frustrated by the lack of help from the medical community.

15. I live in fear that I will become sicker than I am.

16. My doctors don't know how to treat my illness.

17. My doctors believe what I tell them about my symptoms and illness.

18. I have taken matters into my own hands and am treating myself without medical assistance.

19. I have spent thousands of dollars on treatment only to become sicker.

20. I employ stubbornness as a way to combat my disease.

21. I accept my limitations.

22. I accept that things are different.

23. I have made a new life plan since becoming ill.

24. I find support in online groups (e.g., Facebook groups geared to Lyme and chronic illness).

25. I find support in being a member of in-person self-help groups.

26. I spend much of my days on activities focused on symptom management.

27. I have sought out counseling /psychotherapy.

28. I feel like I have been abandoned by friends, family, community, and/or society since becoming ill.

29. I grieve over the loss of my physical health.

30. I grieve over the loss of my independence.

31. I grieve over the loss of my lifestyle change.

32. I feel the losses I have suffered as a result of Lyme disease aren't recognized as grief-worthy because it isn't a death.

33. I feel the losses I have suffered as a result of Lyme disease aren't recognized as grief-worthy because the community at-large does not believe in Post Lyme/Chronic
(85) $94.5 \%$

(4) $4.4 \%$

(1) $1.1 \%$

(41) $45 \%$

(30) $33 \%$

(20) $20 \%$

(76) $83.5 \%$

(11) $12.1 \%$

(4) $4.4 \%$

(86) $95.6 \%$

(4) $4.4 \%$

(0) $0 \%$

(80) $87.9 \%$

(8) $8.8 \%$

(3) $3.3 \%$

(80) $87.9 \%$

(4) $4.4 \%$

(7) $7.7 \%$

(38) $42.2 \%$

(22) $24.4 \%$

(30) $33.4 \%$

(34) $37.8 \%$

(23) $25.6 \%$

(33) $36.6 \%$

(50) $56.2 \%$

(16) $18 \%$

(23) $25.8 \%$

(62) $68.1 \%$

(16) $17.6 \%$

(13) $14.3 \%$

(39) $42.9 \%$

(68) $74.7 \%$

(14) $15.4 \%$

(38) $41.7 \%$

(5) $5.5 \%$

(44) $48.9 \%$

(26) $28.9 \%$

(18) $19.8 \%$

(20) $22.2 \%$

(78) $85.7 \%$

(6) $6.6 \%$

(7) $7.7 \%$

(25) $27.5 \%$

(34) $37.4 \%$

(32) $35.1 \%$

(71) $78 \%$

(11) $12.1 \%$

(9) $9.9 \%$

(47) $50 \%$

(13) $14.4 \%$

(32) $35.6 \%$

(71) $78 \%$

(11) $12.1 \%$

(9) $9.9 \%$

(86) $94.5 \%$

(5) $5.5 \%$

(0) $0 \%$

(77) $84.6 \%$

(11) $12.1 \%$

(3) $3.3 \%$

(83) $91.2 \%$

(76) $83.5 \%$

(5) $5.5 \%$

(3) $3.3 \%$

(7) $7.7 \%$

(8) $8.8 \%$

(79) $86.8 \%$

(7) $7.7 \%$

(5) $5.5 \%$ 
Lyme disease.

34. I don't feel that others are empathic to my situation.

35. I believe that I don't have the right to grieve over my losses I am experiencing and have experienced (The losses in your life as a result of having Lyme disease).
(70) $76.9 \%$

(36) $39.6 \%$
(13) $14.3 \%$

(9) $9.9 \%$
(8) $8.8 \%$

(46) $50.5 \%$

\section{References}

Ali, A., Vitulano, L., Lee, R., Weiss, T,\& Colson, E. (2014). Experiences of patients identifying with chronic Lyme disease in the healthcare system: A qualitative study. Biomed Central Family Practice, 15, doi10.1186/14712296-15-79.

Attig, T. (2004). Disenfranchised grief revisited: Discounting hope and love. Omega, 49(3), 197-215.

Barker, K. (2002). Self-help literature and the making of an illness identity: The case of fibromyalgia syndrome. Social Problems, 49, 279-300.

Boss, P., \& Couden, B. (2002). Ambiguous loss from chronic physical illness: Clinical interventions with individuals, couples, and families. Psychotherapy in Practice, 58(11), 1351-1360.

Cameron, D., Johnson, L., \& Maloney, E. (2014). Evidence assessments and guideline recommendations in Lyme disease: The clinical management of known tick bites, erythema migrans rashes and persistent disease. Retrieved from International Lyme and Associated Diseases Society website: https://www.ilads.org/patientcare/ilads-treatment-guidelines/Center for Disease Control (2019). Data and surveillance. Retrieved from:https://www.cdc.gov/lyme/datasurveillance/index.html?CDC_AA_refVal=https\%3A\%2F\%2Fwww.cdc. gov\%2Flyme\%2Fstats\%2Findex.html

Conrad, P., \& Barker, K. (2010). The social construction of illness: Key insights and policy implications. Journal of Health and Social Behavior, 51 (Suppl.), S67-S79.

Doka, K. (1989). Disenfranchised grief. Lexington, MA: Lexington Books.

Doka, K. (2002). Disenfranchised grief. New directions, challenges, and strategies for practice. Champaign, IL: Research Press.

Drew, D., \& Hewitt, H. (2006). A qualitative approach to understanding patients' diagnosis of Lyme disease. Public Health Nursing, 23, 20-26.

Ferguson, J. (2012). Cure unwanted? Exploring the controversy and why conflicts in practice guidelines may be guiding us down the wrong path. American Journal of Law \& Medicine, 38, 196-224.

Fox, R. (1989). The sociology of medicine: A participant observer's view. Englewood Cliffs, NJ: Prentice Hall.

Gill, P., \& Lowes. L. (2014). Renal transplant failure and disenfranchised grief: Participants' experiences in the first year post-graft failure - a qualitative longitudinal study. International Journal of Nursing Studies, 51, 12711280 .

Hill, D., \& Holmes, T. (2015). Provider knowledge, attitudes, and practices regarding Lyme disease in Arkansas. Journal of Community Health, 40, 339-346.

Horowitz, R. (2017). How can I get better? An action plan for treating resistant Lyme \& chronic disease. New York, New York: St. Martin's Press.

Hyden, L., \& Sachs, L. (1998). Suffering, hope, and diagnosis: On the negotiation of chronic fatigue syndrome. Health, 2, 175-193.

Infectious Diseases Society of America (2019).Retrieved fromhttps://www.idsociety.org/public-health/lymedisease/lyme-disease/International Lyme and Associated Diseases Society. Lyme disease basics for providers. (2019). Retrieved from https://www.ilads.org/research-literature/lyme-disease-basics-for-providers/

Jason, L., \& Reed, J. (2015). The use of mixed methods in studying chronic illness. Health Psychology and Behavioral Medicine, 3(1), 40-51.

Johnson, L., Aylward, A.,\& Stricker, R. (2011). Healthcare access and burden of care for patients with Lyme disease: A large United States survey. Health Policy, 102, 64-71.

Mechanic, D., \& Meyer, S. (2000). Concepts of trust among patients with serious illness. Social Science and Medicine, 51, 657-668.

Morris, R., \& Sanders, C. (2018). Critical moments in long-term condition management: A longitudinal qualitative social network study. Chronic Illness, 14(2), 119-134. 
Nettleton, S., Watt, I., O’Malley, L., \& Duffy, P. (2005). Understanding the narratives of people who live with medically unexplained illness. Patient Education \& Counseling, 56, 205-210.

Nettleton, S. (2006). "I just want permission to be ill::Towards a sociology of medically unexplained symptoms. Social Science \&Medicine, 62, 1167-1178.

Nicolai, J., Muller, N., Noest, S., Gleissner, C., Eich, W., \& Bieber, C. (2018). To change or not to change - That is the question: A qualitative study of lifestyle changes following acute myocardial infraction. Chronic Illness, 14(1), 25-41.

Ortenblad, L., Meillier, L., \& Jonsson, A. (2018). Multi-morbidity: A patient perspective on navigating the health care system and everyday life. Chronic Illness, 14(4), 271-282.

Rebman, A., Aucott, J., Weinstein, E., Bechtold, K., Smith, K.,\& Leonard, L. ( 2017). Living in Limbo: Contested narratives of patients with chronic symptoms following Lyme disease. Qualitative Health Research, 27(4), 534-546.

Siegal, J., Brown, A., Devendoff, A., Collier, J., \& Jason, L. (2018). A content analysis of chronic fatigue syndrome and myalgic encephalomyelitis in the news from 1987-2013. Chronic Illness, 14(1) 3-12.

Slightam, C., Brandt, K., Jenchura, E., Lewis, E., Asch, S, \& Zulman, D. (2018). "I had to change so much in my life to live with my new limitations": Multimorbid patients' descriptions of their most bothersome chronic conditions. Chronic Illness, 14(1), 13-24.

Sobel, S., \& Cowan, C. (2003). Ambiguous loss and disenfranchised grief: The impact of DNA testing on the family system. Family Process, 42(1), 47-57.

Thornton, G., \& Zanich, M. (2002). Empirical assessment of disenfranchised grief: 1989-2000. In Doka, K. (Ed.). Disenfranchised grief: New directions, challenges, and Strategies for practice. Champaign, IL: Research Press. Pp. 79-89.

van den Wijngaard et al. (2015). The burden of Lyme borreliosis expressed in disability-adjusted life years.European Journal of Public Health, 25, 1071-1078.

Wormser et al. (2006). The clinical assessment, treatment, and prevention of Lyme disease, Human Granulocytic, and Anaplasmosis, and Babesiosis: Clinical practice guidelines by the Infectious Diseases Society of America. Clinical Infectious Diseases, 43(9),1089-1134. 